\title{
A Novel Variant of the CHD2 Gene Associated With Developmental Delay and Myoclonic Epilepsy
}

\author{
Lina Zhu ${ }^{1+}$, Fujun Peng ${ }^{2 \dagger}$, Zengwen Deng ${ }^{3}$, Zhichun Feng ${ }^{1}$ and Xiuwei $\mathrm{Ma}^{1 *}$ \\ ${ }^{1}$ Faculty of Pediatrics, Chinese PLA General Hospital, BaYi Children's Hospital, The Seventh Medical Center of PLA General \\ Hospital, Beijing, China, ${ }^{2}$ School of Basic Medical Sciences, Weifang Medical University, Weifang, China, ${ }^{3}$ Hospital of Jvlu \\ County, Xingtai, China
}

OPEN ACCESS

Edited by:

Naglaa M. Kamal,

Cairo University, Egypt

Reviewed by:

Jing Chen,

Cincinnati Children's Hospital Medical

Center, United States

Vanesa Nieto-Estévez,

University of Texas at San Antonio,

United States

Marina Trivisano,

Bambino Gesù Children's Hospital

(IRCCS), Italy

Amy McTague,

University College London,

United Kingdom

*Correspondence:

Xiuwei Ma

pony007@vip.sina.com

${ }^{t}$ These authors have contributed equally to this work and share first

authorship

Specialty section:

This article was submitted to

Genetics of Common and Rare

Diseases,

a section of the journal

Frontiers in Genetics

Received: 19 August 2021

Accepted: 12 January 2022

Published: 11 February 2022

Citation:

Zhu L, Peng F, Deng Z, Feng $Z$ and Ma $X$ (2022) A Novel Variant of the

CHD2 Gene Associated With

Developmental Delay and

Myoclonic Epilepsy.

Front. Genet. 13:761178.

doi: $10.3389 /$ fgene.2022.761178
Pathogenic variants in $C H D 2$ have been reported to have a wide range of phenotypic variability in neurodevelopmental disorders, such as early-onset epileptic encephalopathy, developmental delay, and behavior problems. So far, there is no clear correlation between genotypes and phenotypes. This study reports a Chinese patient with a novel heterozygous CHD2 mutation (c.4318C>T, pArg1440*). Her main clinical manifestations include developmental delay, myoclonic epilepsy, and hypothyroidism. Then, we reviewed a total of 144 individuals carrying $\mathrm{CHD2}$ variants with epileptic encephalopathy. In terms of clinical manifestations, these patients are usually described with variable epilepsy phenotypes, including idiopathic photosensitive occipital epilepsy, Dravet syndrome, Jeavons syndrome, Lennox-Gastaut syndrome, juvenile myoclonic epilepsy, and non-specific epileptic encephalopathy. Among them, myoclonic seizures and generalized tonic-clonic seizures are the main seizure types in all patients hosting $\mathrm{CHD} 2$ single-nucleotide or indel variants (non-CNVs). At the molecular level, there are 102 types of CHD2 non-CNVs in 126 patients, almost one mutational type corresponding to one person, and there is no difference in the incidence ratio of each position. Furthermore, we summarized that a small proportion of patients inherited CHD2 variants, and not all patients with $\mathrm{CHD} 2$ variants had seizures. Importantly, the phenotypes, especially seizures control and fever sensitivity, and genotypes had a relative association. These results enriched the database of CHD2-relative neurodevelopmental disorders and provided a theoretical foundation for researching the relationship between genotypes and phenotypes.

\footnotetext{
Keywords: whole-exome sequencing, CHD2 variants, developmental delay, myoclonic epilepsy, epileptic encephalopathy
}

\section{INTRODUCTION}

Chromodomain helicase DNA-binding protein 2 (CHD2, MIM: 615369) is a member of the CHD family, which is only known to cause the brain-restricted phenotypes when disrupted in humans. It is mapped to chromosome 15q26.1 and is considered an ATP-dependent chromatin remodeling that regulates the transcription expression of many genes (Lamar and Carvill, 2018; Carvill and Mefford, 2015). Some studies have demonstrated that pathogenic variants of the CHD2 gene are associated with childhood-onset developmental and epileptic encephalopathy (DEE), which is a severe form of neurodevelopmental disorder with a wide range of phenotypic variability, including autism spectrum 
disorder (ASD), intellectual disability (ID), developmental delay, microcephalus, behavioral anomalies, facial dysmorphisms, and several types of epilepsy (Carvill and Mefford, 2015; Thomas et al. , 2015; Verhoeven et al., 2016; Chen et al., 2020). Recently, Chen et al. presented the largest single case series of patients with CHD2-related epilepsy and then comprehensively reviewed 53 published cases in the literature through seizure onset age, seizure types, developmental outcome, electroencephalogram (EEG), brain magnetic resonance imaging (MRI), and diagnoses, among others, which improved the understanding on the relationship between genotype and phenotype (Chen et al., 2020). In 2021, De Maria et al. reported 18 new patients and reviewed 84 previously reported patients, getting the results that the median age of seizures onset in $92 \%$ of patients was 2.5 years, and there was no clear association between genotypes and phenotypes (De Maria et al., 2021).

This study reported a novel variant in the CHD2 gene in a Chinese girl with developmental delay and myoclonic epilepsy through whole-exome sequencing (WES). We also systematically reviewed the published literature, provided a thorough overview of clinical, neuroimaging, physical, and genetic findings in CHD2-related epilepsy patients, and built the possible relationship between genotypes and phenotypes.

\section{MATERIALS AND METHODS}

\section{Case Collection}

A total of 59 papers, including 144 cases, were considered candidates (Table 1 and Supplementary Table S1). These cases were collected from studies and databases and reported to associate with the CHD2 mutations. The main process is as follows: 1) the keywords "CHD2" and "neurodevelopmental disorders" or "epileptic encephalopathy" were used to search the PubMed database for relevant papers; 2) the full text of each eligible publication was downloaded and read carefully. The papers and review articles on these cases were saved; 3) using the "CHD2" gene as the keyword, relevant papers in the HGMD database were acquired, downloaded, screened, and reserved, and these papers were associated with CHD2-related epileptic encephalopathy; 4) the collected review articles were used to confirm the conclusions from the obtained papers and complement the lacking literature; 5) patient information, including clinical information, neuroimaging, physical, and genetic findings, was extracted from these papers, such as gender, inheritance, and diagnostic findings; and 6) all results were checked by more than two researchers, and the opposite consequences were verified after discussion.

\section{Whole-Exome Sequencing}

Genomic DNA was extracted from the peripheral blood of all the family members using DNA Isolation Kit (Blood DNA Kit V2, CW2553) according to standard procedure. Whole-exome capture and sequencing were performed using SureSelect Human All Exon V6 $(60 \mathrm{Mb})$ kit (Agilent, Santa Clara, USA, and sequenced on the Illumina Nova series platform (Illumina, San Diego, USA), generating 150 bp paired-end pairs. The raw reads of sequencing underwent the process of trimming, depolluting, and filtering to get only the high-quality reads. Only those that passed these filtrations could be used for the downstream analyses. High-quality paired-end reads were aligned to the human reference genome sequence from the UCSC database (build 37.1 version hg19, http://genome.ucsc. edu/) using the Burrows-Wheeler Alignment tool. We estimated quality scores and made the consensus SNP and insertions and deletions (indels) calling using GATK [1]. All the called variants were annotated with ANNOVAR software to give the variant position, variant type, allele frequency, conservation prediction, and so forth, which would help locate mutations relative to diseases. All variants were filtered using the 1000 Genomes, ExAC, ChES, and gnomAD databases, as well as a minor allele frequency $(\mathrm{MAF}) \leq 1 \%$. Then, a series of analyses were used with OMIM database, HGMD database, and phenotypes-genotypes association analysis. Finally, the candidate variants were judged according to the ACMG standard and validated by Sanger sequencing. All samples were obtained with written informed consent from patients.

\section{Statistical Analyses}

All the statistical analyses were performed using SPSS 22.0. Pearson $\chi^{2}$ test was used to assess the significance of differences between groups. In the study of the number of patients with various seizure types (Table $\mathbf{2}$ and Supplementary Table S2), the average value was introduced, which was equal to the number of all the patients except for unclassified generalized seizures (GS), unclassified seizures, and not applicable (NA) divided by the number of seizure types containing at least one patient. Similarly, the average concept was introduced in the study of the number of patients with various epilepsy types/syndrome (Figure 3 and Supplementary Table S3). In the study of the incidence rate of variants in different domains of CHD2 (Figure 4A and Supplementary Table S4), the incidence rate was calculated by dividing the mutational numbers in one domain divided by mRNA sequence lengths of this domain, and the average value was equal to the number of all the variants divided by the length of mRNA sequence in $\mathrm{CHD} 2$ gene. In the studies of seizures control, photosensitivity, and fever sensitivity (Figures 4B,C and Supplementary Table S5), the difference values were presented among different domains of CHD2. A two-sided $p$-value $<0.05$ was considered statistically significant and was adjusted by the Bonferroni correction.

\section{Clinical Report}

The proband was a 3-year and 2-month old girl, who was a fullterm baby born by a healthy and unrelated Chinese couple through cesarean section, weighing $3,900 \mathrm{~g}$, without intrauterine distress, suffocation, and neonatal jaundice (Figure 1A). Three days after birth, a thyroid function test suggested hypothyroidism, and the child took thyroxine tablets regularly to maintain normal thyroid function. She started to roll over at 6 months and sit at 8 months. At 17 months, she could not walk alone, and her Development Screen Test (DST) presented $\mathrm{DQ}<70$ and $\mathrm{MI}<70$. The DQ scores of Gesell Developmental Assessment (GDA) are listed below: gross movement is 71, fine 
TABLE 1 | The seizure types of patients with CHD2 mutations.

Single-nucleotide or indel

variants $(n=126)$

Gender
Age
Inheritance
Age of seizure onset
Development before seizure onset
Seizure control
Introduce factors
Photosensitivity
Fever sensitivity
Cognition/development outcome
ASD
ADHD
ID
Behavior problem
Other abnormal findings ${ }^{\#}$
Physical development
Height
Weight
Head circumference
EEG
MRI
Facial dysmorphisms
$\mathrm{F}(43), \mathrm{M}(61), \mathrm{NA}(22)$
$0 \sim 6$ years (26), 6 12 years (27), >12 years (37), NA (36)
De novo (111), mother (2), father (3), NA (10)
$\leq 1$ year (16), 1 6 years (70), 6 12 years (5), >12 years (2), NA (33)
Delay (47), normal (22), NA (57)
Yes (18), no (36), NA (72)
Yes (45), no (37), NA (44)
Yes (14), no (37), NA (75)
Yes (34), no (48), NA (44)
Yes (12), no (48), NA (66)
Yes (76), no (8), NA (42)
Yes (47), no (2), NA (77)
Yes (78), no (8), NA (40)
Normal (17), short stature (6), tall stature (1), NA (102)
Normal (11), underweight (5), obesity (2), NA (108)
Normal (15), microcephaly (9), NA (102)
Normal (2), abnormal (80), NA (44)
Normal (64), abnormal (8), NA (54)
yes (11), no (7), NA (108)

Copy number variants $(n=$

18)
$\mathrm{F}(11), \mathrm{M}(7)$

0 6 years (3), 6 12 years (4), >12 years (8), NA (3)

de novo (18), mother (0), father (0), NA (0)

$\leq 1$ year (2), 1 6 years (11), 6 12 years (3), >12 years (1), NA (1)

Delay (12), normal (0), NA (6)

Yes (6), no (9), NA (3)

Yes (5), no (1), NA (12)

Yes (0), no (4), NA (14)

Yes (7), no (5), NA (6)

Yes (1), no (8), NA (9)

Yes (15), no (3), NA (0)

Yes (10), no (0), NA (8)

Yes (16), no (0), NA (2)

Normal (4), short stature (6), tall stature (0), NA (8)

Normal (6), underweight (1), obesity (2), NA (9)

Normal (2), microcephaly (3), NA (13)

Normal (0), abnormal (9), NA (9)

Normal (9), abnormal (5), NA (4)

Yes (12), no (0), NA (6)

Abbreviations: $d$, day; $m$, month; $y$, year; $A D H D$, attention deficit hyperactivity disorder; ASD, autism spectrum disorder; EEG, electroencephalography; $F$, female; ID, intellectual disability; M, male; MRI, magnetic resonance imaging; NA, not applicable; \#, other abnormal findings mainly contained delay in motor and language development, learning disability, illusions or hearing odd sounds, defective social communications, poor balance, short-term memory problems, etc.

movement is 76 , adaptive skills score is 71 , language is 53 , and social score is 71 . Brain magnetic resonance imaging (MRI) revealed no severe abnormalities. Then, the patient was required to do a series of rehabilitation training, which resulted in her ability to walk alone at 1.5 years, and run and navigate stairs at 2.5 years. However, she still could not jump or execute instructions and presented poor balance. The development delay was shown in her intelligence and language; for example, she spoke a single word at 2 years and said two or three words at 3 years. She liked to run around and open her mouth and did not like to play with other children. At the age of 2 years and 8 months, she started to experience seizures, and there was no obvious cause such as photosensitivity and fever sensitivity. The seizures were manifested by a rapid shaking of the entire body, which relieved in 1-2 s and occurred several times per day. After remission, the mental reaction was as usual. There was no special family history. The video electroencephalogram (VEEG) showed high-amplitude spike waves, and slow spike waves were burst throughout the brain, especially in the frontal, central, and middle posterior temporal regions (Figures 2A,B). When she had seizures, VEEG showed a generalized polyspike wave (Figure 2C). Other auxiliary examinations results were normal and mainly contained electrocardiogram, blood routine, urine routine, stool routine, biochemistry, myocardial enzymes, thyroid function, blood ammonia, lactic acid, blood tandem mass spectrometry analysis of amino acids and acylcarnitine, and urine organic acid analysis. Oral anti-epileptic treatment with sodium valproate was gradually increased to $30 \mathrm{mg} / \mathrm{kg} \cdot \mathrm{d}$, and seizure was controlled.
The girl came to our hospital because of mental retardation with seizures when she was 3 years and 2 months old. She presented normal facial features, active behavior, poor execution, and lacking eye contact with people. The physical examination results revealed a total length of $96 \mathrm{~cm}$, weight of $15 \mathrm{~kg}$, and head circumference of $49 \mathrm{~cm}$. The cardiopulmonary and abdominal examinations showed no abnormality. Dystonia of limbs did not exist, sputum culture reflexes were drawn symmetrically, and pathological signs were negative. The second DQ scores were a gross movement of 60, fine movement of 69 , adaptive skills of 66 , language of 50 , and social score of 67. Brainstem auditory evoked response (BAER) showed a delay in the peripheral conduction of the binaural auditory pathway. Visual evoked potential (VEP) showed that the incubation period of bilateral P100 was prolonged. Integrating the above information, the girl was diagnosed with developmental delay and myoclonic epilepsy.

\section{Genetic Analysis}

Whole-exome sequencing was performed on the proband and her parents. Overall, more than $8 \mathrm{~Gb}$ of the sequence generated per patient covered all the exome and splice site regions of genes. The proband revealed a de novo heterozygous variant in CHD2 (c. $4318 \mathrm{C}>\mathrm{T}, \mathrm{p} . \operatorname{Arg} 1440^{*}$ ). This nonsense variant was localized in the 30th exon out of 39 total exons in the CHD2 gene (NM_001271.4) and was predicted to cause premature termination of translation. In addition, the mutation does not exist in the 1000 Genomes, ExAC, ChES, and gnomAD databases (Macdonald et al., 2014; Lek et al., 2016), and to our knowledge, it has not been reported in affected individuals in the published literature. 


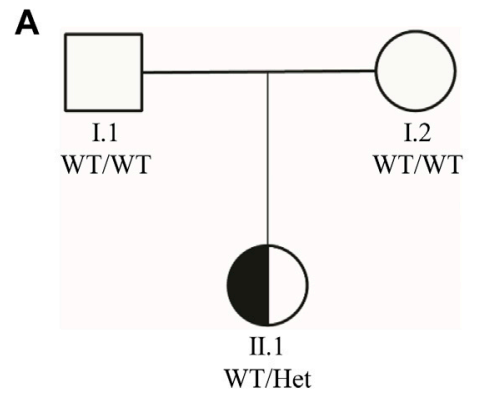

C

Human AKSSSKSKRSQ'GPVHITAG Mouse GKSSSKSKRSOGPVHITAG

Rat AKSSSKSKRSOGPVHITAG

Chicken AKSGSKGKRSQGPVHITAG

Bovin AKSSSKSKRSO్1GPVHITAG

Chimpanzee AKSSSKSKRSOGPVHITAG

Horse AKSSSKSKRSO్AGPVHITAG

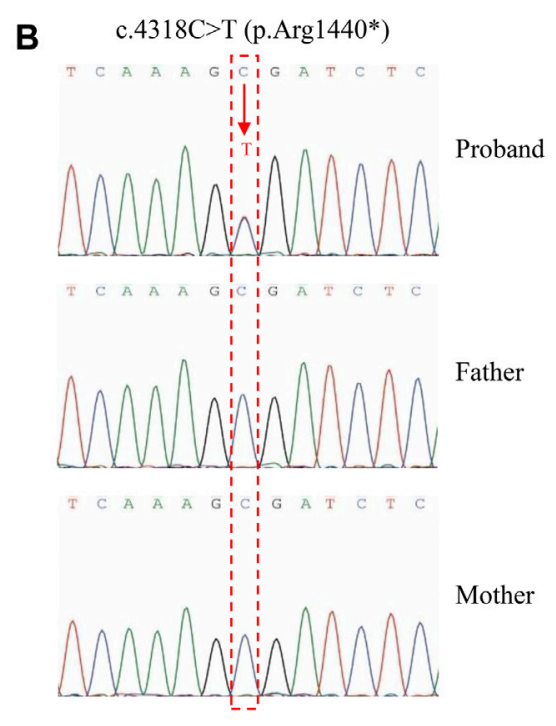

B

FIGURE 1 | Genetic characterization of the proband. (A) Family pedigree. (B) Analysis of CHD2 c.4318C>T (NM_001271.4, p. Arg1440*) in a non-related Chinese family. (C) Homology alignment of the protein encoded by CHD2.

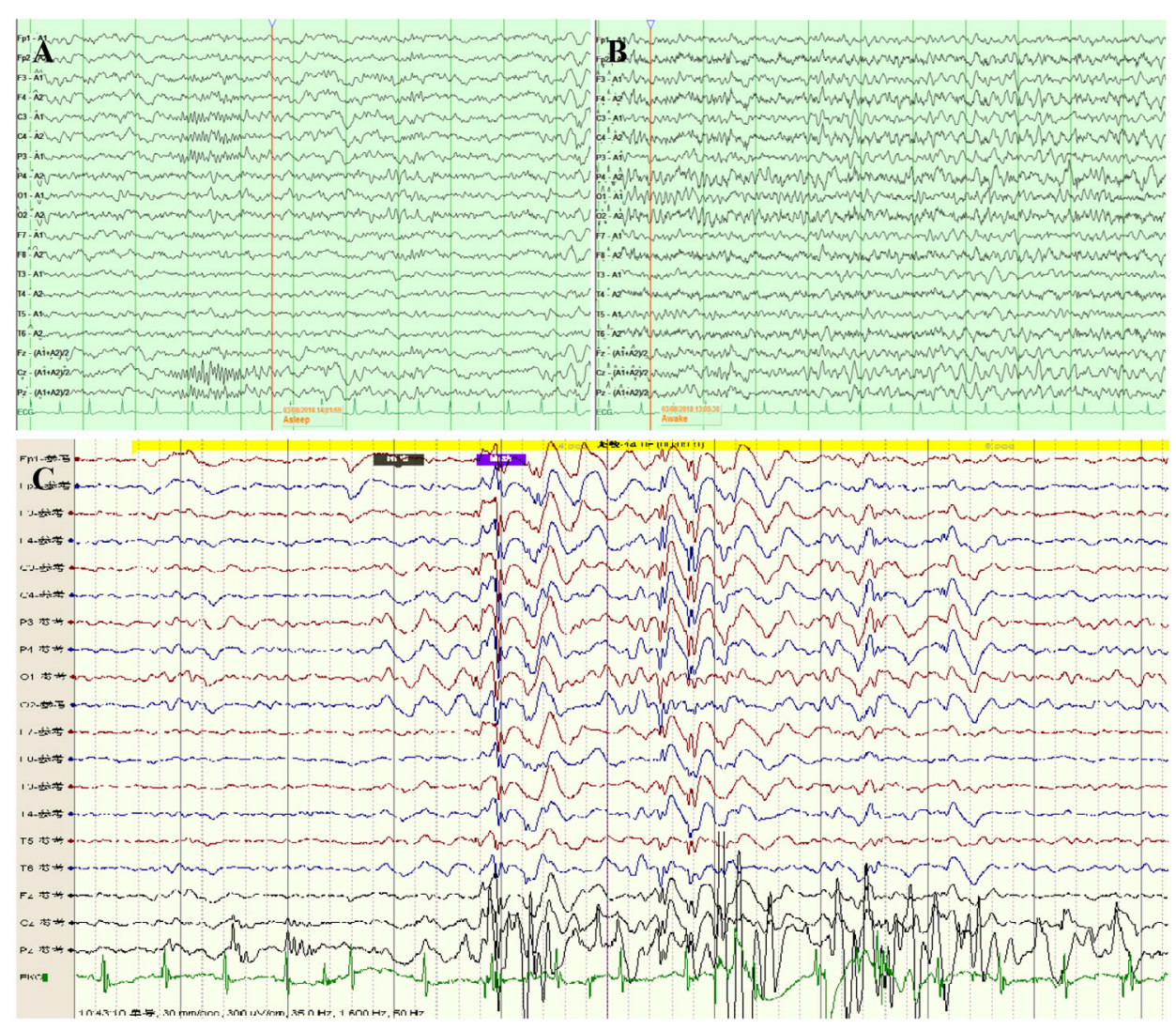

FIGURE 2 | Video electroencephalogram (VEEG) of the proband with development disorders. (A) During the sleeping period, a small amount of medium amplitude sharp waves and sharp slow waves were emitted asynchronously in bilateral frontal, central, parietal, and right occipital regions, and sporadic in the right anterior temporal region. (B) During the awake period, there had 7-8 $\mathrm{Hz}$ low amplitude, a, and $\Theta$ mixed waves in the bilateral occipital area. (C) When she had myoclonic seizures, the results showed a generalized polyspike wave. 
TABLE 2 | Clinical characteristics of persons with a CHD2 mutation in published cases.

\begin{tabular}{|c|c|c|c|c|c|c|}
\hline \multirow[b]{2}{*}{ Seizure types } & \multicolumn{3}{|c|}{ Single-nucleotide or indel variants } & \multicolumn{3}{|c|}{ Copy number variants } \\
\hline & First & Further & All & First & Further & All \\
\hline aAS & 3 & $11^{b}$ & $14^{b}$ & 0 & 0 & 0 \\
\hline AbS & 5 & $13^{b}$ & $18^{b}$ & 5 & 0 & 5 \\
\hline aMAS & $0^{a}$ & 0 & $0^{\mathrm{a}}$ & 1 & 0 & 1 \\
\hline AtS & 4 & $15^{\mathrm{b}}$ & $19^{b}$ & 0 & 0 & 0 \\
\hline CSE & 2 & 3 & 5 & 0 & 0 & 0 \\
\hline DA & $0^{\mathrm{a}}$ & 1 & 1 & 0 & 0 & 0 \\
\hline EMA & 8 & 4 & $12^{b}$ & 1 & 1 & 2 \\
\hline EMs & $0^{a}$ & 1 & 1 & 0 & 1 & 1 \\
\hline ES & 2 & 0 & 2 & 0 & 0 & 0 \\
\hline FCS & $0^{a}$ & 0 & $0^{a}$ & 0 & 1 & 1 \\
\hline FoS & 3 & $15^{\mathrm{b}}$ & $18^{b}$ & 1 & 0 & 1 \\
\hline FoSID & 1 & 0 & 1 & 1 & 0 & 1 \\
\hline FS & 14 & 5 & $19^{b}$ & 3 & 0 & 3 \\
\hline GCS & $0^{a}$ & 0 & $0^{\mathrm{a}}$ & 0 & 1 & 1 \\
\hline GTCS & 17 & $30^{b}$ & $47^{\mathrm{b}}$ & 2 & $5^{\star}$ & $6^{*}$ \\
\hline $\mathrm{HD}$ & $0^{a}$ & 2 & 2 & 0 & 1 & 1 \\
\hline MA & 1 & 6 & 7 & 0 & 0 & 0 \\
\hline MAS & 3 & 8 & 10 & 0 & 0 & 0 \\
\hline MCS & $0^{\mathrm{a}}$ & 1 & 1 & 0 & 0 & 0 \\
\hline MS & $24^{b}$ & $30^{b}$ & $52^{\mathrm{b}}$ & 0 & 4 & 4 \\
\hline NCSE & $0^{a}$ & 10 & 10 & 0 & 1 & 1 \\
\hline NS & $0^{a}$ & 1 & 1 & 0 & 0 & 0 \\
\hline SE & $0^{a}$ & 8 & 8 & 0 & 0 & 0 \\
\hline TCS & 5 & 7 & 12 & 0 & 0 & 0 \\
\hline TS & 1 & $11^{b}$ & 12 & 0 & 2 & 2 \\
\hline Unclassified GS & 4 & 0 & 4 & 3 & 1 & 3 \\
\hline Unclassified seizures & 33 & 0 & 28 & 1 & 0 & 1 \\
\hline $\mathrm{NA}$ & 1 & 67 & 0 & 0 & 10 & 0 \\
\hline
\end{tabular}

Abbreviations: aAS, atypical absence seizures; AbS, absence seizures; aMAS, atypical myoclonic-absence seizures; AtS, atonic seizures; CNVs, copy number variants; CSE, convulsive status epilepticus; DA, drop attack; EMA, eyelid myoclonia with absence; EMs, eyelid myoclonias; ES, epileptic spasm; FCS, febrile clonic seizures; FoS, focal seizures; FoSID, focal seizure with impairment of awareness; FS, febrile seizures; GCS, generalized clonic seizure; GS, generalized seizures; GTCS, generalized tonic-clonic seizures; HD, head drops; MA, myoclonic absence seizures; MAS, myoclonic-atonic seizures; MCS, myotonic-clonic seizures; MS, myoclonic seizures; NA, not applicable; NCSE, non-convulsive status epilepticus; NS, nonepileptic seizures; SE, status epilepticus; TCS, tonic-clonic seizures; TS, tonic seizures; *, the difference did not exist using the Bonferroni correction.

atendence and p-value $<0.05$;

${ }^{b}$ resistance and p-value $<0.05$.

This variant was also validated by Sanger sequencing. Meanwhile, the CHD2 genes of her parents were normal (Figures 1A,B). No other significant variants were found in the other genes. Multiple sequence alignment analysis revealed that the p. Arg1440* substitution occurred on an amino acid residue which was evolutionarily highly conserved among different species (Figure 1C).

\section{DISCUSSION}

The CHD protein family (containing CHD1-CHD9) is mainly involved in the ATP-dependent chromatin remodelers that contribute to the reorganization of chromatin structure and deposition of histone variants necessary for regulating gene transcription expression. Among the nine CHD family members, the CHD2 pathogenic variants only lead to a brainrestricted phenotype when disrupted in humans, which indicates a unique role for this gene in neurodevelopment (Lamar and Carvill, 2018).

CHD2 mutation-related epilepsy was first published in 2009 (Veredice et al., 2009). This paper described a 30-month-old girl with refractory myoclonic epilepsy associated with mental retardation, growth delay, peculiar facial appearance, minor physical anomalies, and photosensitivity. In the patient, a 15q26.1-15q26.2 deletion including CHD2 was detected by CGH-array. To date, there are 144 reported individuals encompassing 126 single-nucleotide or indel variants (nonCNVs) and 18 copy number variants (CNVs) with CHD2related epileptic encephalopathy (Table 1 and Supplementary Table S1). From Table 1 and Supplementary Table S1, almost all patients were diagnosed at over 1 year or even over 6 years of age. Up to now, most CHD2 variants have been reported de novo in patients with epilepsy rather than in an inherited mode. Nevertheless, in 2015, the first patients diagnosed with eyelid myoclonia with absence (EMA) inherited it from an unaffected mother, who carried the CHD2 variant c.653C > T (p. Pro218Leu) (Galizia et al., 2015). In 2018, the patient who inherited a pathogenic CHD2 variant c.628G $>$ T (p. Glu218*) from the affected mother was published (Petersen et al., 2018). The distressing thing is that compared with her daughter's refractory epilepsy, her seizures have always been well controlled (Petersen et al., 2018). In 2020, Chen et al. reported 


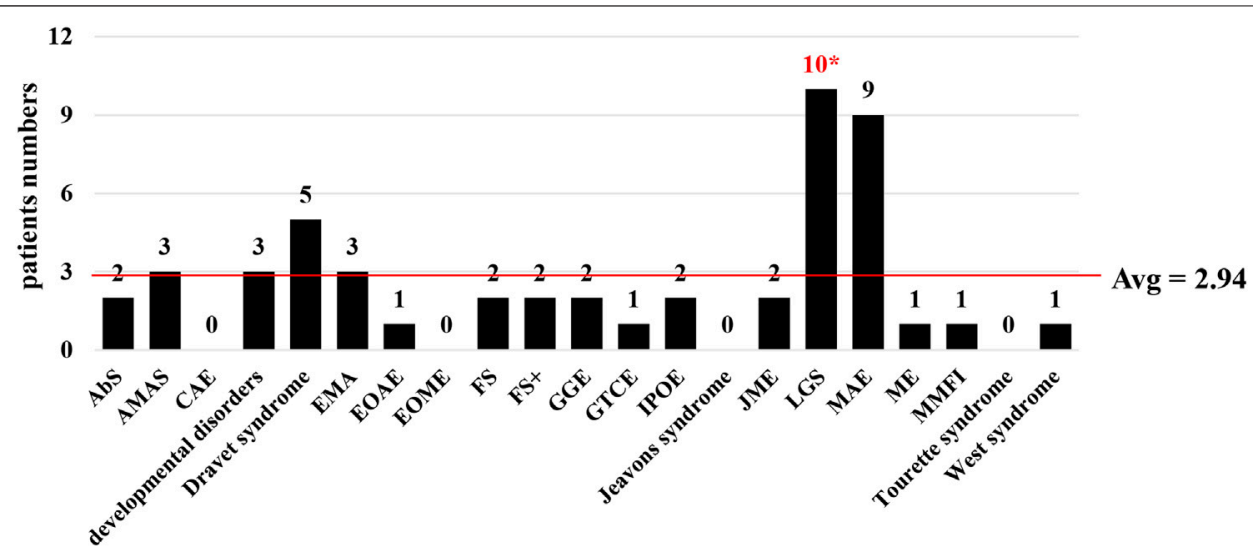

FIGURE 3 | Statistical analysis of all reported CHD2-non-CNVs patients with epilepsy. Distributions of patients according to epilepsy syndrome in the overall cohort, including 126 non-CNVs patients. The epilepsy syndrome did not contain DEE, non-specific GE, non-specific EE, and unclassified epilepsy. The average value = patients' numbers of epilepsy syndrome/numbers of epilepsy types. Abbreviations: AbS, absence seizures; CAE, childhood absence epilepsy; DEE, developmental and epileptic encephalopathy; EE, epileptic encephalopathy; EMA, eyelid myoclonia with absence; EOAE, early-onset absence epilepsy; EOME, early-onset myoclonic epilepsy; FS, febrile seizures; FS+, febrile seizure plus; GE, generalized epilepsy; GGE, genetic generalized epilepsy; GTCE, generalized tonic-clonic epilepsy; IPOE, idiopathic photosensitive occipital epilepsy; JME, juvenile myoclonic epilepsy; LGS, Lennox-Gastaut syndrome; MAE, myoclonic atonic epilepsy; ME, myoclonic epilepsy; MMFI, malignant migrating focal seizures in infancy; non-CNVs, single-nucleotide or indel variants; ${ }^{*}$, the difference did not exist using the Bonferroni correction.

a sporadic case inherited from an unaffected father with c. $5153+2 \mathrm{~T}>\mathrm{C}$ variant of CHD2 gene and dizygotic twins inherited from the affected father with c.5232G $>$ A (p. Met1744Ile) (Chen et al., 2020). To our surprise, the five mutations in patients did not occur again in other cases. In CHD2 CNVs patients, the inherited patients were not reported. These findings indicate that CHD2 variants, with the exception of CHD2 CNVs, can be inherited (Table 1).

Previously, CHD2 variants were generally considered to be childhood-onset epileptic encephalopathy. However, more and more individuals with CHD2 mutants were published, and the ages of seizure onset were different from infancy to childhood (Table 1 and Supplementary Table S1). We had also found that different patients had different development in pre-seizure onset. For example, Carvill et al. reported that two out of six patients with epileptic encephalopathies caused by $\mathrm{CHD} 2$ mutations had normal development and another four were abnormal (Carvill et al., 2013). Interestingly, all the patients with CHD2 CNVs could lead to developmental delays in pre-seizure onsets, such as motor and speech developmental delays (Table 1 and Supplementary Table S1). In addition, the inducing factors, including photosensitivity and fever sensitivity, EEG results, and facial dysmorphisms, were also various among CHD2 variants' patients. Galizia et al. performed photosensitivity tests on zebrafish larvae with $C H D 2$ gene knockout and found that the CHD2 gene knockout significantly enhanced the photosensitivity of congenital zebrafish larvae. Their results had confirmed that this gene was related to photosensitive epilepsy (Galizia et al., 2015). Except for the twin patients mentioned above, most patients had abnormal EEG results (Chen et al., 2020). We also found that the reported patients had behavior problems such as aggressive behavior. In other aspects of patients, the findings showed diversity, such as ASD, height, and MRI (Table 1 and Supplementary Table S1).

The patients have variable seizure types, including absence seizures (AbS), atonic seizures, tonic-clonic seizures, and myoclonic absence seizures. Importantly, an individual can have multiple seizure types; even the first seizure and the later seizure could be of different types (Supplementary Table S1). Based on these phenomena, we summarized and analyzed the seizure types of patients with CHD2 non-CNVs and CNVs (Table 2 and Supplementary Table S2). In the CHD2 nonCNVs patients, both the myoclonic seizures (MS, 27.3\%, 24/ 88 ) and generalized tonic-clonic seizures (GTCS, 19.3\%, 17/ 88 ) were the top two types of the first seizures, which presented the difference than average value (5.87 people/seizure types). Some seizure types, including atypical myoclonic-absence seizures (aMAS, $0 \%$ ) and febrile clonic seizures (FCS, 0\%), did not exist. In contrast, MS (50.8\%, 30/59), GTCS (50.8\%, 30/ 59 ), atonic seizures (AtS, 25.4\%, 15/59), focal seizures (FoS, $25.4 \%, 15 / 59)$ and andother three seizure types were the main types of second and later seizures compared to average value (2.95 people/seizure types). In general, MS, GTCS, AtS, febrile seizures (FS), absence seizures (AbS), FoS, atypical absence seizures (aAS), and eyelid myoclonia with absence (EMA) were the main seizure types in CHD2 non-CNVs (Table 2 and Supplementary Table S2). Interestingly, the main type for CHD2 CNVs patients could be GTCS, which did not exist using the Bonferroni correction. This phenomenon could be related to the small samples. Furthermore, the patients' numbers in different epileptic encephalopathy were analyzed using the above methods (Figure $\mathbf{3}$ and Supplementary Table S3). The results showed that Lennox-Gastaut syndrome (LGS) was usually diagnosed in 

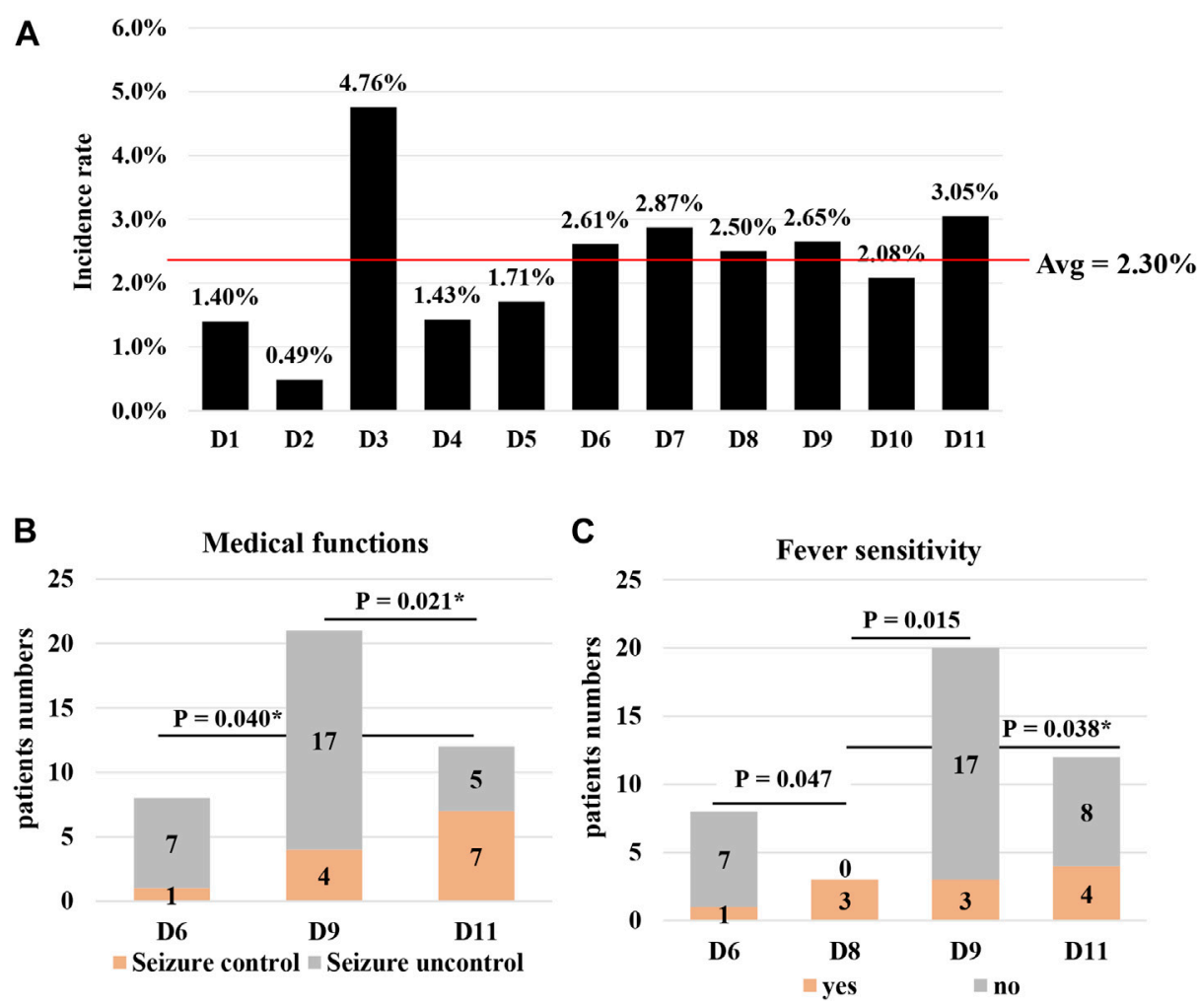

FIGURE 4 | Association analysis between genotypes and phenotypes in CHD2 non-CNVs patients. (A) Distributions of the incidence rate of 126 non-CNVs in different $\mathrm{CHD} 2$ domains. The incidence rate was the mutational numbers in one domain divided by mRNA sequence lengths of this domain and the average value equal to all the variants numbers divided by mRNA sequence lengths of the CHD2 gene. (B,C) The association analysis between CHD2 genotypes in different domains and phenotypes including seizure control and fever sensitivity in CHD2 non-CNVs patients. Abbreviations: non-CNVs, single-nucleotide or indel variants; reference protein sequences, NP_001262.3; *, the difference did not exist using Bonferroni correction; D1, 1-262aa; D2, 263-330aa, chromodomain; D3, 331-337aa; D4, 378447aa, chromodomain; D5, 448-486aa; D6, 487-767aa, SNF2 family; D7, 768-825aa; D8, 826-905aa, helicase conserved C-terminal domain; D9, 906-1458aa; D10, 1459-1554aa, domain of unknown function; D11, 1555-1828aa.

CHD2 non-CNVs patients, which presented the difference compared to the average value (2.94 people/epileptic encephalopathy) using the Pearson $\chi 2$ test, and the adjusted difference with the Bonferroni correction was disappearance (Supplementary Table S3). The CHD2 CNVs individuals did not exist in these results (Supplementary Table S3).

Though a series of studies indicated that there was no clear correlation between genotypes and phenotype in CHD2related epileptic encephalopathy (Epi et al., 2013; Chen et al., 2020; De Maria et al., 2021), we thought that it was necessary to further research the association between genotypes and phenotypes. We referred to the mRNA sequences NM_001271.4 and protein sequences NP_001262. 3 to analyze all the CHD2 non-CNVs related epilepsy patients (Thomas et al., 2015; Carvill and Mefford, 2015) (Figure 4 and Supplementary Tables S4, S5). The whole protein sequences were divided into 11 fragments (D1-D11), including four conserved domains (Thomas et al., 2015). There were 102 types of CHD2 non-CNVs in 126 patients, and almost one mutational type corresponded to one patient. Among them, the c.4173dupA (p. Gln1392Thrfs ${ }^{\star} 17$ ) and c.5035C>T (p.
Arg1679*) could be the hotspot variants but only occurred five times, respectively (Supplementary Table S4). The CHD2 variants were distributed in the whole protein sequences and focused on the D9 fragments (906-1458aa, 33.92\%, 37/126) (Supplementary Table S4), which led to a serious shortcoming because the longer the domain, the more the number. In order to address this shortcoming, the incidence rate was applied. Though the D11 fragments (1554-1828aa, 3.05\%, 25/825) tended to host the CHD2 variants, the difference value was not shown than the average value $(2.30 \%)$ (Figure $4 \mathrm{~A}$ and Supplementary Table S4). Meanwhile, The D3 fragment was too short and was not further analyzed. Interestingly, the incidence rate presented the difference between D1 and D11 (Supplementary Table S4). We also analyzed the relationship between $C H D 2$ genotypes and seizure control, inducing factors including photosensitivity and fever sensitivity in all the CHD2 non-CNVs patients, and found a significant correlation between genotypes and phenotypes (Figures 4B,C and Supplementary Table S5). The patients with CHD2 variants in D11 tended to have better seizure control, and patients with CHD2 variants in D6 and D9 tended to have worse seizure 
control. Similarly, there also was a difference in fever sensitivity among D6, D8, D9, and D11. The patients in D8 tended to have more serious fever sensitivity compared to D6, D9, and D11. Though the difference for photosensitivity did not present with $C H D 2$ variants in $\mathrm{D} 6, \mathrm{D} 9$, and D11, patients with $C H D 2$ variants in D9 suffered photosensitivity more easily than CHD2 variants in D6 and D11. These results suggested that there could be associations between genotypes and phenotypes, which were further demonstrated by the dizygotic or monozygotic twins (Pinto et al., 2016; Wang et al., 2017; Chen et al., 2020). Pinto et al. reported that the monozygosity cases had the same seizure onset at 2 years and 6 months old, carried the c.4173dupA (p. Gln1392Thrfs $\left.{ }^{\star} 17\right)$ mutations, and presented a set of similar clinical features phenotypes, including autism spectrum disorder, hypotonia, postnatal microcephaly, stereotypic movements, circadian rhythm alterations, and AbS (Pinto et al., 2016). Wang et al. also reported that the monozygotic twins had the same mutation c.5035C $>\mathrm{T}$ (p. Arg1679*) and showed similar clinical features (Wang et al., 2017). In another paper, the dizygotic twins were diagnosed with febrile seizure plus (FS+), carried the c.5232G $>$ A (p. Met1744Ile) mutation inherited from the affected father, showed similar clinical descriptions such as fever introduce, MRI normal, EEG normal, first seizure type GTCS, and seizure control except for seizure onset and further seizure type (Chen et al., 2020).

A large number of studies demonstrated the phenotypic heterogeneity of CHD2-associated epilepsy. So far, 144 patients have been reported to have epileptic seizures and associated CHD2 variants (Table 1 and Supplementary Table S1). However, not all patients with $C H D 2$ variants have seizures. Up to now, a total of 9 patients with $C H D 2$ variants were reported to have no epilepsy (Bhakta et al., 2005; Kulkarni et al., 2008; Chenier et al., 2014; Hamdan et al., 2014; Pinto et al., 2014; Chen et al., 2017; Cabrera-Salcedo et al., 2019), For example, Hamdan et al. found that a severe ID girl with motor and speech development delays and CHD2 variant c.335C $>$ G (p. Ser112*) did not have the history of epilepsy (Hamdan et al., 2014). Interestingly, patients without epilepsy tended to be CHD2 CNVs patients (Bhakta et al., 2005; Kulkarni et al., 2008; Chenier et al., 2014; Hamdan et al., 2014; Pinto et al., 2014). So far, five patients carrying CHD2 CNVs but without epilepsy have been reported. For example, Pinto et al. reported a patient with a de novo deletion in the CHD2 gene, with ASD, mild ID, and dysmorphic features including micrognathia and protruding ears, but no seizures (Pinto et al., 2014). However, his ASD-affected brother carried the same deletion of the CHD2 gene, had similar dysmorphic features and mild ID, and experienced an epilepsy onset at 9 years of age. Kulkarni et al. described a de novo translocation $\mathrm{t}(\mathrm{X} ; 15)(\mathrm{p} 22.2 ; \mathrm{q} 26.1)$ dn disrupting CHD2 in a child with developmental delay, scoliosis, and hirsutism (Kulkarni et al., 2008).

According to the data, seizures in about $1 / 4$ of patients are controlled through single or multiple anti-epileptic drugs (Table 1 and Supplementary Table S1). Interestingly, the ages of seizure onset of patients who were seizures control and carried CHD2 non-CNVs were from 1 to 12 years, and generalized tonic- clonic seizures (GTCS, 61.1\%, 11/18) and myoclonic seizures (MS, 38.9\%, 7/18) were the top two seizure types (Supplementary Table S1). However, most patients with CHD2-related neurodevelopmental disorders remain refractory to treatment. Although in some epilepsy syndromes, a ketogenic diet could have vital benefits (Daci et al., 2018; Zhou et al., 2018), a total of four patients with $C H D 2$ variants were treated with the ketogenic diet, which did not produce significant effects (Thomas et al., 2015; Costain et al., 2019). Therefore, a large sample size and follow-up studies would be helpful to define the treatment and prognosis of CHD2-related epilepsy.

\section{CONCLUSION}

In this study, a girl with developmental delay and myoclonic epilepsy caused by a new mutation c.4318C > T (pArg1440*) in the CHD2 gene was studied using WES. The mutation produced a nonsense variant and disrupted the CHD2 protein structure.

Subsequently, all patients with reported neurodevelopmental disorder and variants in the CHD2 gene were systematically reviewed and analyzed. A total of 144 patients, including 126 non-CNVs and 18 CNVs with CHD2 pathogenic variants, were analyzed. We found that, except for $\mathrm{CHD} 2 \mathrm{CNV}$, a small portion of patients obtained $\mathrm{CHD} 2$ variants by inheritance rather than de novo. The ages of seizure onset with CHD2 mutants varied from infancy to childhood, even adults. The patients with CHD2 non-CNVs had a certain tendency toward the seizure types, such as MS and GTCS. Meanwhile, not all patients with CHD2 variants had seizures. Although almost one mutational type corresponded to one person, there may be an association between genotypes and phenotypes, especially for seizure control and fever sensitivity. The above results can provide a theoretical basis for better research on CHD2-related neurodevelopmental disorders.

\section{DATA AVAILABILITY STATEMENT}

The datasets for this article are not publicly available due to concerns regarding participant/patient anonymity. Requests to access the datasets should be directed to the corresponding author.

\section{ETHICS STATEMENT}

The studies involving human participants were reviewed and approved by the Ethical Committee of the Seventh Medical Center of PLA General Hospital. Written informed consent to participate in this study was provided by the participant's legal guardian/next of kin. Written informed consent was obtained from the individual(s), and minor(s)' legal guardian/next of kin for the publication of any potentially identifiable images or data included in this article. 


\section{AUTHOR CONTRIBUTIONS}

LZ, FP, and XM conducted and designed the experiment. LZ and FP wrote the manuscript, performed the experiment, and analyzed the data. LZ and ZD collected the samples. ZF and $\mathrm{XM}$ were responsible for the supervision and coordination of the study process. All authors approved the final manuscript as submitted and agreed to be accountable for all aspects of the work.

\section{FUNDING}

All phases of this study were supported by the Seventh Medical Center of PLA General Hospital. This project has received funding from the National Key Research and Development

\section{REFERENCES}

Bhakta, K. Y., Marlin, S. J., Shen, J. J., and Fernandes, C. J. (2005). Terminal Deletion of Chromosome 15q26.1: Case Report and Brief Literature Review. J. Perinatol 25, 429-432. doi:10.1038/sj.jp.7211301

Cabrera-Salcedo, C., Hawkes, C. P., Tyzinski, L., Andrew, M., Labilloy, G., Campos, D., et al. (2019). The GenomicsTargeted Searches of the Electronic Health Record and Genomics Identify an Etiology in Three Patients with Short Stature and High IGF-I Levels. Horm. Res. Paediatr. 92, 186-195. doi:10.1159/ 000504884

Carvill, G. L., Heavin, S. B., Yendle, S. C., Mcmahon, J. M., O'roak, B. J., Cook, J., et al. (2013). Targeted Resequencing in Epileptic Encephalopathies Identifies De Novo Mutations in CHD2 and SYNGAP1. Nat. Genet. 45, 825-830. doi:10. 1038/ng.2646

Carvill, G. L., Mefford, H. C., Adam, M. P., Ardinger, H. H., Pagon, R. A., Wallace, S. E., et al. (2015). "CHD2-Related Neurodevelopmental Disorders," in GeneReviews((R)) (Seattle (WA). [updated 2021 Jan 21]).

Chen, C.-P., Chen, C.-Y., Chern, S.-R., Wu, P.-S., Chen, S.-W., Lai, S.-T., et al. (2017). Molecular Cytogenetic Characterization of a Duplication of 15q24.2q26.2 Associated with Anencephaly and Neural Tube Defect. Taiwanese J. Obstet. Gynecol. 56, 550-553. doi:10.1016/j.tjog.2017.06.003

Chen, J., Zhang, J., Liu, A., Zhang, L., Li, H., Zeng, Q., et al. (2020). CHD2 -related Epilepsy: Novel Mutations and New Phenotypes. Dev. Med. Child. Neurol. 62, 647-653. doi:10.1111/dmcn.14367

Chénier, S., Yoon, G., Argiropoulos, B., Lauzon, J., Laframboise, R., Ahn, J. W., et al. (2014). CHD2 Haploinsufficiency Is Associated with Developmental Delay, Intellectual Disability, Epilepsy and Neurobehavioural Problems. J. Neurodevelop Disord. 6, 9. doi:10.1186/1866-1955-6-9

Costain, G., Cordeiro, D., Matviychuk, D., and Mercimek-Andrews, S. (2019). Clinical Application of Targeted Next-Generation Sequencing Panels and Whole Exome Sequencing in Childhood Epilepsy. Neuroscience 418, 291-310. doi:10.1016/j.neuroscience.2019.08.016

Daci, A., Bozalija, A., Jashari, F., and Krasniqi, S. (2018). Individualizing Treatment Approaches for Epileptic Patients with Glucose Transporter Type1 (GLUT-1) Deficiency. Int. J. Mol. Sci. 19. doi:10.3390/ijms19010122

De Maria, B., Balestrini, S., Mei, D., Melani, F., Pellacani, S., Pisano, T., et al. (2021). Expanding the Genetic and Phenotypic Spectrum of CHD2-Related Disease: From Early Neurodevelopmental Disorders to Adult-Onset Epilepsy. Am. J. Med. Genet. A. 188 (2), 522-533. doi:10.1002/ajmg.a.62548

Epi, K. C., Allen, A. S., Berkovic, S. F., Cossette, P., Delanty, N., Dlugos, D., et al. (2013). De Novo mutations in Epileptic Encephalopathies. Nature 501, 217-221. doi:10.1038/nature12439

Galizia, E. C., Myers, C. T., Leu, C., De Kovel, C. G. F., Afrikanova, T., CorderoMaldonado, M. L., et al. (2015). CHD2variants Are a Risk Factor for Photosensitivity in Epilepsy. Brain 138, 1198-1208. doi:10.1093/brain/awv052
Projects (2016YFC1000707), Weifang Medical College Doctoral Start Fund (2020BSQD40), and Natural Science Foundation of Shandong Province (ZR2021QH367).

\section{ACKNOWLEDGMENTS}

The authors would thank the patients for their participation in this study and the teachers for providing critical advice in data analysis.

\section{SUPPLEMENTARY MATERIAL}

The Supplementary Material for this article can be found online at: https://www.frontiersin.org/articles/10.3389/fgene.2022.761178/ full\#supplementary-material

Hamdan, F. F., Srour, M., Capo-Chichi, J.-M., Daoud, H., Nassif, C., Patry, L., et al. (2014). De Novo mutations in Moderate or Severe Intellectual Disability. Plos Genet. 10, e1004772. doi:10.1371/journal.pgen.1004772

Kulkarni, S., Nagarajan, P., Wall, J., Donovan, D. J., Donell, R. L., Ligon, A. H., et al. (2008). Disruption of Chromodomain Helicase DNA Binding Protein 2 (CHD2) Causes Scoliosis. Am. J. Med. Genet. 146A, 1117-1127. doi:10.1002/ ajmg.a. 32178

Lamar, K.-M. J., and Carvill, G. L. (2018). Chromatin Remodeling Proteins in Epilepsy: Lessons from CHD2-Associated Epilepsy. Front. Mol. Neurosci. 11, 208. doi:10.3389/fnmol.2018.00208

Lek, M., Karczewski, K. J., Karczewski, K. J., Minikel, E. V., Samocha, K. E., Banks, E., et al. (2016). Analysis of Protein-Coding Genetic Variation in 60,706 Humans. Nature 536, 285-291. doi:10.1038/nature19057

Macdonald, J. R., Ziman, R., Yuen, R. K. C., Feuk, L., and Scherer, S. W. (2014). The Database of Genomic Variants: a Curated Collection of Structural Variation in the Human Genome. Nucl. Acids Res. 42, D986-D992. doi:10. 1093/nar/gkt958

Petersen, A. K., Streff, H., Tokita, M., and Bostwick, B. L. (2018). The First Reported Case of an Inherited Pathogenic CHD2 Variant in a Clinically Affected Mother and Daughter. Am. J. Med. Genet. 176, 1667-1669. doi:10. 1002/ajmg.a. 38835

Pinto, A. M., Bianciardi, L., Mencarelli, M. A., Imperatore, V., Di Marco, C., Furini, S., et al. (2016). Exome Sequencing Analysis in a Pair of Monozygotic Twins Re-evaluates the Genetics behind Their Intellectual Disability and Reveals a CHD2 Mutation. Brain Dev. 38, 590-596. doi:10. 1016/j.braindev.2015.12.006

Pinto, D., Delaby, E., Merico, D., Barbosa, M., Merikangas, A., Klei, L., et al. (2014). Convergence of Genes and Cellular Pathways Dysregulated in Autism Spectrum Disorders. Am. J. Hum. Genet. 94, 677-694. doi:10.1016/j.ajhg. 2014.03.018

Thomas, R. H., Zhang, L. M., Carvill, G. L., Archer, J. S., Heavin, S. B., Mandelstam, S. A., et al. (2015). CHD2 Myoclonic Encephalopathy Is Frequently Associated with Self-Induced Seizures. Neurology 84, 951-958. doi:10.1212/wnl. 0000000000001305

Veredice, C., Bianco, F., Contaldo, I., Orteschi, D., Stefanini, M. C., Battaglia, D., et al. (2009). Early Onset Myoclonic Epilepsy and 15q26 Microdeletion: Observation of the First Case. Epilepsia 50, 1810-1815. doi:10.1111/j.15281167.2009.02078.x

Verhoeven, W., Egger, J., Knegt, A., Zuydam, J., and Kleefstra, T. (2016). Absence Epilepsy and the CHD2 Gene: an Adolescent Male with Moderate Intellectual Disability, Short-Lasting Psychoses, and an Interstitial Deletion in 15q26.1-q26.2. Ndt 12, 1135-1139. doi:10.2147/ndt.s102272

Wang, Y., Du, X., Bin, R., Yu, S., Xia, Z., Zheng, G., et al. (2017). Genetic Variants Identified from Epilepsy of Unknown Etiology in Chinese Children by Targeted Exome Sequencing. Sci. Rep. 7, 40319. doi:10. 1038/srep40319 
Zhou, P., He, N., Zhang, J.-W., Lin, Z.-J., Wang, J., Yan, L.-M., et al. (2018). Novel Mutations and Phenotypes of Epilepsy-Associated Genes in Epileptic Encephalopathies. Genes, Brain Behav. 17, e12456. doi:10.1111/gbb.12456

Conflict of Interest: The authors declare that the research was conducted in the absence of any commercial or financial relationships that could be construed as a potential conflict of interest.

Publisher's Note: All claims expressed in this article are solely those of the authors and do not necessarily represent those of their affiliated organizations, or those of the publisher, the editors, and the reviewers. Any product that may be evaluated in this article, or claim that may be made by its manufacturer, is not guaranteed or endorsed by the publisher.

Copyright $\odot 2022 \mathrm{Zhu}$, Peng, Deng, Feng and Ma. This is an open-access article distributed under the terms of the Creative Commons Attribution License (CC BY). The use, distribution or reproduction in other forums is permitted, provided the original author(s) and the copyright owner(s) are credited and that the original publication in this journal is cited, in accordance with accepted academic practice. No use, distribution or reproduction is permitted which does not comply with these terms. 